\title{
Efficacy and tolerability of renzapride in irritable bowel syndrome: a meta-analysis of randomized, controlled clinical trials including 2528 patients
}

Shilan Mozaffari ${ }^{1}$, Shekoufeh Nikfar ${ }^{2,3}$, Mohammad Abdollahi ${ }^{1,2}$

\begin{abstract}
'Department of Toxicology and Pharmacology, Faculty of Pharmacy, Tehran University of Medical Sciences, Tehran, Iran

2Department of Pharmacoeconomics and Pharmaceutical Administration, Faculty of Pharmacy, Tehran University of Medical Sciences, Tehran, Iran ${ }^{3}$ Iranian Food and Drug Administration, Ministry of Health and Medical Education, Tehran, Iran
\end{abstract}

Submitted: 5 June 2013

Accepted: 18 August 2013

Arch Med Sci 2014; 10, 1: 10-18

DOI: 10.5114 /aoms.2014.40729

Copyright @ 2014 Termedia \& Banach

\section{Abstract}

Introduction: By targeting different subtypes of 5-hydroxytryptamine $(5 \mathrm{HT})$ receptors in the gastrointestinal (GI) tract, several drugs have been introduced for the management of irritable bowel syndrome (IBS). Renzapride is a full agonist for 5HT4 receptor and an antagonist to 5HT2 $\mathrm{b}$ and $5 \mathrm{HT} 3$ receptors which is thought a promising therapeutic agent for constipation predominant IBS (C-IBS) patients due to its accelerating effect on the GI tract. In this meta-analysis, our aim was to evaluate the efficacy and tolerability of renzapride in the management of IBS.

Material and methods: A search was done from 1992 to February 2013 for placebo-controlled trials that investigated the efficacy of renzapride in IBS. Results: Relative risk (RR) for clinical efficacy in IBS patients treated for 5 weeks or less comparing renzapride to placebo was $1.07(95 \% \mathrm{Cl}=0.89-$ $1.29, p=0.38)$. This value for IBS patients treated for more than 5 weeks was $1.04(95 \% \mathrm{Cl}=0.78-1.239, p=0.77)$. The RR for clinical efficacy in IBS patients treated with renzapride $(4 \mathrm{mg})$ for 5 weeks or less and more than 5 weeks in comparison to placebo was $1.2(95 \% \mathrm{Cl}=0.97-1.48, p=0.1)$ and $1.16(95 \% \mathrm{Cl}=0.98-1.37, p=0.08)$, respectively, which were statistically non-significant but clinically important. The analysis of tolerability demonstrated that amongst different reported adverse effects, renzapride caused diarrhea more than placebo $(\mathrm{RR}=1.61$ with a $95 \% \mathrm{Cl}=1.16-2.24$, $p=0.004)$. The RR for withdrawals from renzapride compared to placebo was $1.58(95 \% \mathrm{Cl}=1.26-2.07, p=0.0007)$.

Conclusions: Renzapride is not superior to placebo in relieving IBS symptoms and causes significant incidences of diarrhea and drop-outs due to adverse effects in treated patients vs. placebo. Thus, this medicine might be a cost burden to patients without providing good effectiveness.

Key words: renzapride, 5-hydroxytryptamine, irritable bowel syndrome, clinical trial, meta-analysis, systematic review.

\section{Introduction}

Irritable bowel syndrome (IBS) is a highly prevalent chronic functional disorder of the gastrointestinal (Gl) tract which has different prospects

\author{
Corresponding author: \\ Prof. Mohammad Abdollahi \\ PharmD, PhD, FIAS \\ Faculty of Pharmacy \\ and Pharmaceutical \\ Sciences Research Center \\ Tehran University \\ of Medical Sciences \\ Tehran 1417614411, Iran \\ Phone: + 982164122319 \\ Fax: + 982166959104 \\ E-mail: \\ Mohammad.Abdollahi@ \\ UToronto.Ca, \\ Mohammad@TUMS.Ac.Ir
}


in patients. Besides predominant symptoms such as abdominal pain and bloating, altered bowel habits classify the syndrome as diarrhea, constipation or alternative predominant types. Several pathophysiological mechanisms have been identified in IBS such as genetics, visceral hypersensitivity, GI motility dysfunction, inflammation and altered bowel microbial flora, and imbalance in secretion of 5-hydroxytryptamine (5HT) [1, 2]. Forasmuch as the exact cause of this disorder is not fully understood, management of IBS is limited to relieving the main symptoms in patients. Fiber, antispasmodics [3], tricyclic antidepressants [4], serotonin reuptake inhibitors [5], antibiotics [6], probiotics [7] and several herbal preparations [8], besides cognitive behavioral therapy [9], are currently administered alone or in combination by physicians. Other types of medicines which have been introduced are 5HT receptor modulators. The $5 \mathrm{HT}$ secreted from enterochromaffin cells regulates the $\mathrm{Gl}$ tract motility. By targeting different subtypes of receptors ( $5 \mathrm{HT} 1,5 \mathrm{HT} 3,5 \mathrm{HT} 4$ ), several drugs have been introduced such as alosetron, cilansetron (5HT3 antagonist) and tegaserod (5HT4 partial agonist) [10, 11]. Prucalopride, mosapride (5HT4 agonist) and ramosetron (5HT3 antagonist) are novel substances under evaluation in phase III of a clinical trial; we have recently reviewed their efficacy, safety and pharmacokinetic issues [12].

Renzapride (a novel benzamide substitute) is a full agonist for $5 \mathrm{HT} 4$ receptor and an antagonist to $5 \mathrm{HT} 2 \mathrm{~b}$ and $5 \mathrm{HT} 3$ receptors. By stimulating $5 \mathrm{HT} 4$ and $5 \mathrm{HT} 2 \mathrm{~b}$ receptors, it accelerates the $\mathrm{Gl}$ tract transit and motility [13]. It is thought a promising therapeutic agent for constipation predominant IBS (C-IBS) patients. Forasmuch as pharmacokinetic and safety studies demonstrated its well tolerability with less adverse effects [14, 15], several clinical trials have been performed to evaluate its potential efficacy in IBS patients. Several clinical trials have confirmed that renzapride does not cause cardiac arrhythmias in clinical dosages, unlike cisapride $[14,15]$. Renzapride is excreted renally and it is not metabolized by cytochrome P450 enzymes. Thus no drug interactions via affecting cytochrome P450 enzymes have been reported $[13,15]$. Due to its demonstrated prokinetic property, most trials have shown that renzapride increases colonic transit and reduces transit time and pain in IBS patients which is beneficial especially in those with constipation [16]. In addition, in dose finding studies, a dose-dependent relation has been observed for its effects [15]. In a meta-analysis performed in 2009, renzapride showed no benefit in IBS patients [17].

In the present meta-analysis, the updated results of clinical trials on efficacy and tolerability of renzapride in IBS patients have been evaluated.

\section{Material and methods}

Renzapride $\left(\mathrm{C}_{16} \mathrm{H}_{22} \mathrm{ClN}_{3} \mathrm{O}_{2}\right)$ molecular formula and structure was presented in Figure 1.

\section{Data sources}

We searched PubMed, Google Scholar, Web of Science, Scopus, and Cochrane Central Register of Controlled trials for placebo-controlled trials that investigated the efficacy of renzapride in IBS management. Data were collected from 1992 to February 2013. The search terms were "renzapride", "5-hydroxytryptamine", "irritable bowel syndrome”, "constipation”, "functional bowel disease" and "irritable colon". All published studies as well as abstracts presented at the meetings were evaluated without any language limitations. The reference list from retrieved articles was reviewed for additional applicable studies.

\section{Study selection}

Two reviewers independently examined the title and abstract of each article to eliminate duplicates, reviews, case studies, pilot studies and uncontrolled trials. All studies investigating effects of renzapride in IBS patients compared to placebo were considered. Trials were disqualified if their outcomes did not have any relation to clinical improvement. We included studies that used Rome criteria for IBS diagnosis. The reviewers independently extracted data on patient's characteristics, sample size, dosage, trial duration, and outcome measures. There were no disagreements between reviewers.

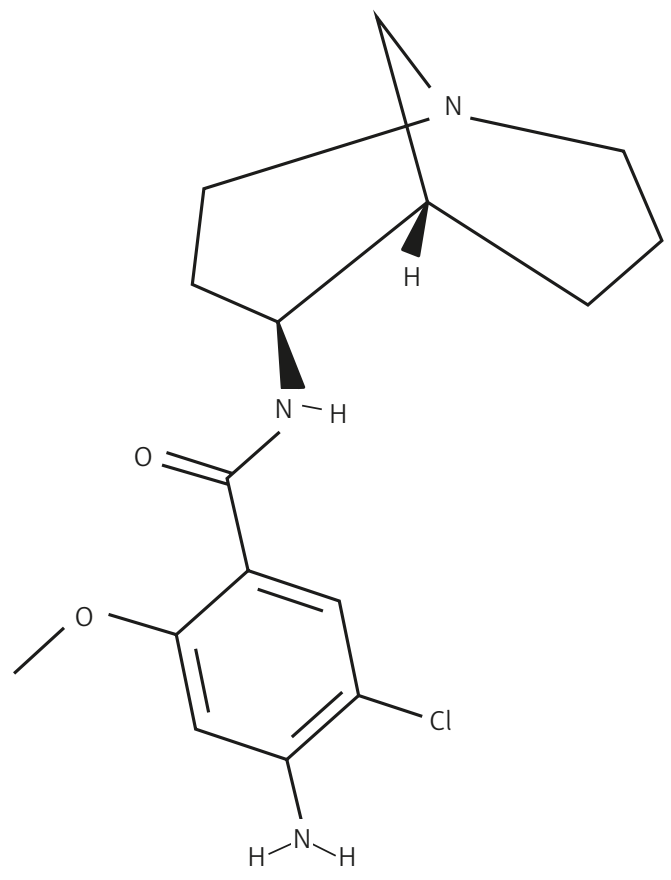

Figure 1. Molecular formula and structure of renzapride 

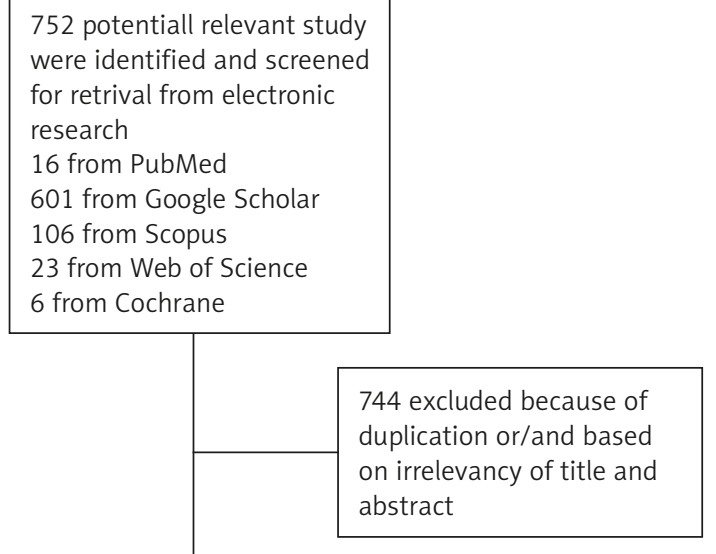

6 reports retrived

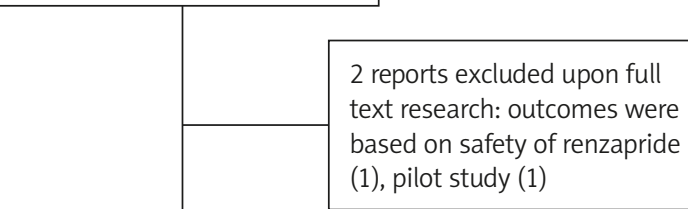

4 eligible randomized controlled trials were included in meta-analysis

Figure 2. Flow diagram of the study selection process

\section{Assessment of trial quality}

Quality of studies was rated by use of the Jadad score, which is based on adherence of studies to randomization, blinding, and dropouts (withdrawals) [18]. The quality scale ranges from 0 to 5 points with a low quality report of score 2 or less and a high quality report of score at least 3.

\section{Statistical analysis}

Data from selected studies were extracted in the form of $2 \times 2$ tables by study characteristics. Included studies were weighted and pooled. Data were analyzed using StatsDirect software version 2.7.9. Relative risk (RR) and $95 \%$ confidence intervals $(95 \% \mathrm{Cl})$ were calculated using Mantel-Haenszel, Rothman-Boice (for fixed effects) or Der Simonian-Laird (for random effects) methods. The Cochran $\mathrm{Q}$ test was used to test heterogeneity and $p<0.05$ was considered significant. In case of heterogeneity or few included studies, the random effects model was used. Funnel plot was used as a publication bias indicator. Clinical importance was evaluated by the Edwards-Nunnally method.

\section{Results}

The electronic searches yielded 752 items: 16 from PubMed, 601 from Google Scholar, 106 from Scopus, 23 from Web of Science and 6 from the Cochrane Central Register of Controlled Trials. Of these, six were scrutinized in full text, and four were considered eligible, had a well-defined global response outcome and were included in this analysis (Figure 2). Two of the studies had a quality score of $4[19,20]$ and the two other studies had a score of 3 [21, 22] (Table I). These four trials included 2528 patients randomized to receive either renzapride or placebo. Of the total, 2421 (95.77\%) were women and 107 (4.23\%) were men. In three of the trials C-IBS patients (meeting the Rome criteria) were involved $[19,21,22]$ and in one trial non C-, non D-IBS patients were involved [20]. Patients' characteristics, type, and dosage of renzapride and placebo, duration of treatment, and outcomes (clinical improvement and the relief of abdominal pain and discomfort) for each study are shown in Tables II and III. Different adverse events of renzapride compared to placebo in IBS patients are summarized in Table IV.

\section{Effectiveness}

Clinical efficacy of renzapride in comparison to placebo in irritable bowel syndrome patients for 5 weeks or less than 5 weeks therapy

The summary of RR for clinical efficacy in IBS patients treated for 5 weeks or less in 3 included trials comparing renzapride to placebo [20-22] was 1.07 with $95 \% \mathrm{Cl}=0.89$ to $1.29(p=0.38$, Figure $3 \mathrm{~A}$ ). The Cochrane $\mathrm{Q}$ test for heterogeneity indicated that the studies are not heterogeneous ( $p=0.51$, Figure 3 B) and could be combined but because of few included studies the random effects for individual and summary of RR was applied. Regression of normalized effect vs. precision for all included studies for clinical efficacy in IBS patients treated for 5 weeks or less with

Table I. Quality score of randomized controlled trials included in the meta-analysis

\begin{tabular}{|lcccc|}
\hline Study & Randomization & Blinding & $\begin{array}{c}\text { Withdrawals } \\
\text { and dropouts }\end{array}$ & Total score \\
\hline George et al. 2008 & 1 & 1 & 1 & 3 \\
\hline Lembo et al. 2010 & 2 & 1 & 1 & 4 \\
\hline Camilleri et al. 2004 & 1 & 1 & 1 & 3 \\
\hline Spiller et al. 2008 & 2 & 1 & 1 & 4 \\
\hline
\end{tabular}


renzapride vs. placebo could not be calculated because of too few strata.

\section{Clinical efficacy of renzapride in comparison to placebo in irritable bowel syndrome patients for more than 5 weeks therapy}

The summary of RR for clinical efficacy in IBS patients treated for more than 5 weeks in 3 included trials comparing renzapride to placebo $[19,20,22]$ was 1.04 with $95 \% \mathrm{Cl}=0.78$ to 1.239 $(p=0.77$, Figure $4 \mathrm{~A})$. The Cochrane $\mathrm{Q}$ test for heterogeneity indicated that the studies are heterogeneous ( $p=0.004$, Figure 4 B) and could not be combined; thus the random effects for individual and summary of RR was applied. Regression of normalized effect vs. precision for all included studies for clinical efficacy in IBS patients treated for more than 5 weeks with renzapride vs. placebo could not be calculated because of too few strata.

\section{Clinical efficacy of renzapride $4 \mathrm{mg}$ daily in comparison to placebo in irritable bowel syndrome patients for 5 weeks or less than 5 weeks therapy}

The summary of RR for clinical efficacy in IBS patients treated for 5 weeks or less in 3 included trials comparing renzapride to placebo [20-22] was 1.2 with $95 \% \mathrm{Cl}=0.97$ to 1.48 ( $p=0.1$, Figure $5 \mathrm{~A}$ ), a statistically non-significant result but clinically important. The Cochrane Q test for heterogeneity indicated that the studies are not heterogeneous ( $p=0.37$, Figure 5 B) and could be combined but because of few included studies the random effects for individual and summary of RR was applied. Regression of normalized effect vs. precision for all included studies for clinical efficacy in IBS patients treated for 5 weeks or less with renzapride $4 \mathrm{mg}$ daily vs. placebo could not be calculated because of too few strata.

\section{Clinical efficacy of renzapride $4 \mathrm{mg}$ daily in comparison to placebo in irritable bowel syndrome patients for more than 5 weeks therapy}

The summary of RR for clinical efficacy in IBS patients treated for more than 5 weeks in 3 included trials comparing renzapride to placebo $[19,20$, 22] was 1.16 with $95 \% \mathrm{Cl}=0.98$ to 1.37 ( $p=0.08$, Figure 6 A), a statistically non-significant result but clinically important. The Cochrane Q test for heterogeneity indicated that the studies are heterogeneous ( $p=0.2$, Figure 6 B) and could be combined but because of few included trials the random effects for individual and summary of RR was applied. Regression of normalized effect vs. precision for all included studies for clinical efficacy in IBS patients

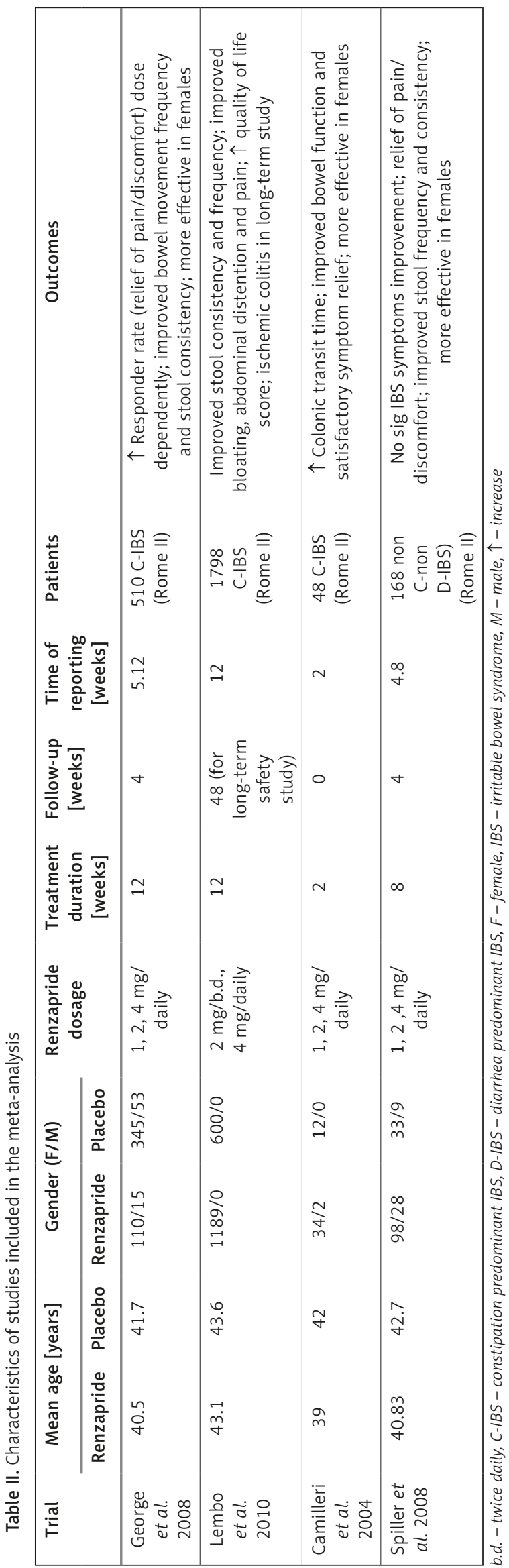


Table III. Clinical improvement (IBS relief) in included studies

\begin{tabular}{|lcccc|}
\hline Study & \multicolumn{3}{c|}{ Time of reporting (duration) } \\
\cline { 2 - 5 } & \multicolumn{2}{c|}{$\leq \mathbf{5}$ weeks } & \multicolumn{2}{c|}{$>$ 5 weeks } \\
\cline { 2 - 5 } & Renzapride & Placebo & $190 / 384$ & $71 / 125$ \\
\hline George et al. 2008 & $188 / 384$ & $58 / 125$ & $378 / 1198$ & $146 / 600$ \\
\hline Lembo et al. 2010 & - & - & - & - \\
\hline Camilleri et al. 2004 & $15 / 36$ & $2 / 11$ & $68 / 126$ & $23 / 42$ \\
\hline Spiller et al. 2008 & $57 / 126$ & $18 / 42$ & \\
\hline
\end{tabular}

Table IV. Different adverse events of renzapride compared to placebo in IBS patients

\begin{tabular}{|lccccc|}
\hline Adverse events & $\begin{array}{c}\text { RR combined } \\
\text { meta-analysis }\end{array}$ & $95 \% \mathrm{Cl}$ & $\begin{array}{c}\text { Value of } p \text { for } \\
\text { RR }\end{array}$ & $\begin{array}{c}\text { Value of } p \text { for } \\
\text { heterogeneity }\end{array}$ & $\begin{array}{c}\text { Publication } \\
\text { bias }\end{array}$ \\
\hline Diarrhea & 1.61 & $1.16-2.24$ & 0.004 & 0.28 & No \\
\hline Headache & 1.21 & $0.93-1.56$ & 0.16 & 0.39 & 0.51 \\
\hline Abdominal pain & 1.37 & $0.95-1.98$ & 0.09 & 0.98 & NA \\
\hline Constipation (aggravated) & 0.98 & $0.55-1.74$ & 0.94 & 0.27 & NA \\
\hline Nausea & 1.27 & $0.76-2.11$ & 0.36 & 0.96 & 0.75 \\
\hline Dyspepsia (gas) & 0.94 & $0.45-1.96$ & 0.47 & NA \\
\hline Vomiting & 1.2 & $0.74-1.95$ & & No \\
\hline
\end{tabular}

NA - not applicable

A

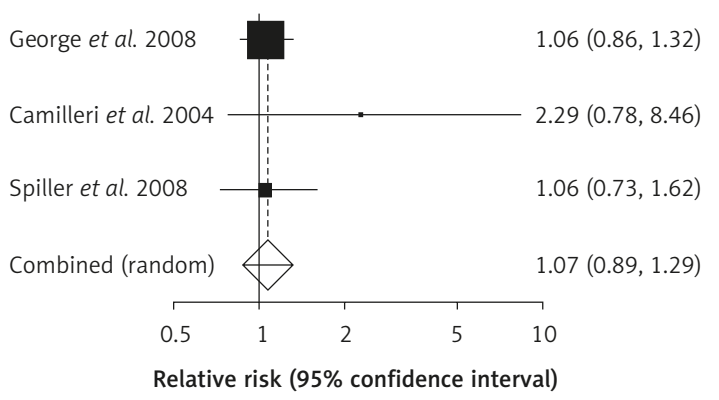

B

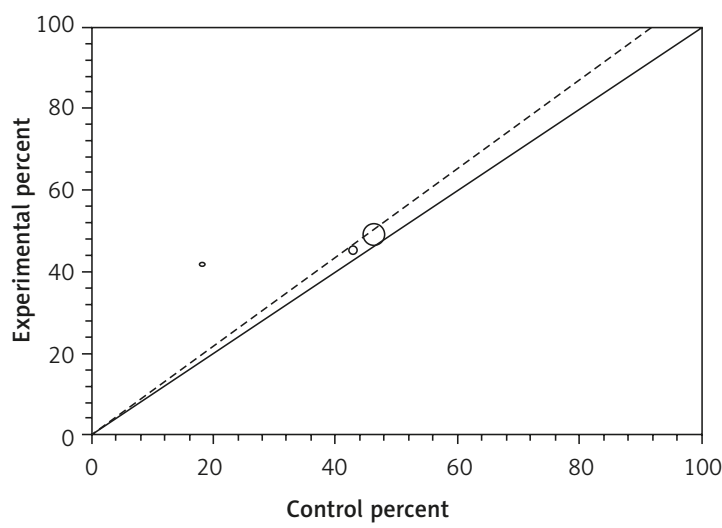

Figure 3. A - Individual and pooled relative risk for the outcome of "clinical efficacy treated for 5 weeks or less" in the studies considering renzapride compared to placebo therapy in IBS patients. B - Heterogeneity indicators for the outcome of "clinical efficacy treated for 5 weeks or less" in the studies considering renzapride compared to placebo therapy in IBS patients

treated for more than 5 weeks with renzapride $4 \mathrm{mg}$ daily vs. placebo could not be calculated because of too few strata.

\section{Tolerability}

Different adverse events of renzapride compared to placebo in irritable bowel syndrome patients

The summary RR for diarrhea in 4 trials [19-22] was 1.61 with a $95 \% \mathrm{Cl}$ of $1.16-2.24$ and a signifi- cant RR $(p=0.004)$. The Cochrane $\mathrm{Q}$ test for heterogeneity indicated that the studies are homogeneous $(p=0.28)$ and could be combined; thus the fixed effects for individual and summary of RR was applied.

The summary RR for headache in 4 trials [1922] was 1.21 with a $95 \% \mathrm{Cl}$ of $0.93-1.56$ and a non-significant RR $(p=0.16)$. The Cochrane Q test for heterogeneity indicated that the studies are not heterogeneous $(p=0.39)$ and could be combined; thus the fixed effects for individual and summary of RR was applied. 
A

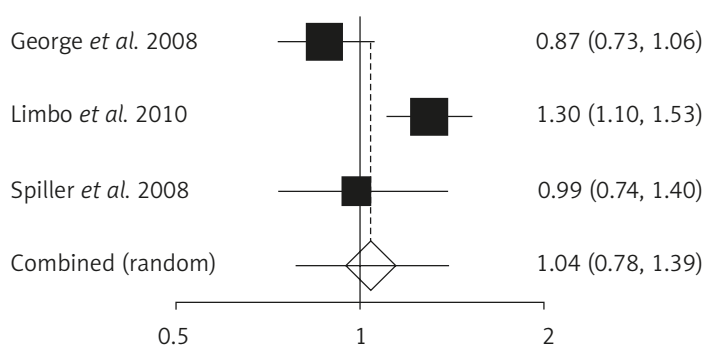

Relative risk ( $95 \%$ confidence interval)
B

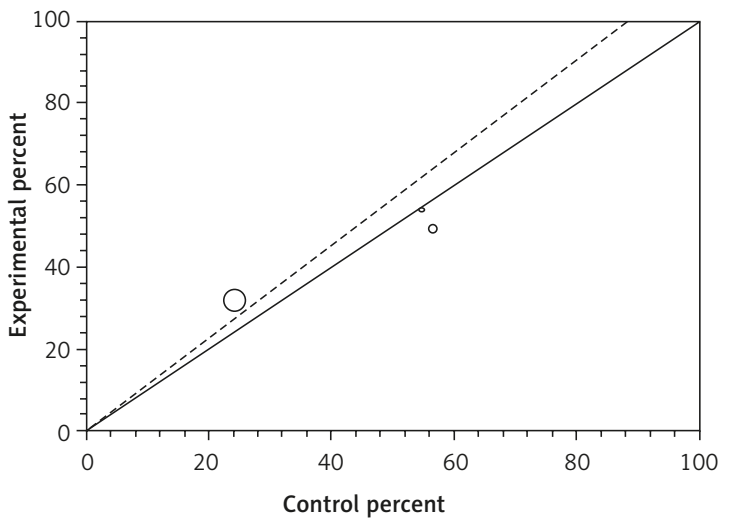

Figure 4. A - Individual and pooled relative risk for the outcome of "clinical efficacy treated for more than 5 weeks" in the studies considering renzapride compared to placebo therapy in IBS patients. B - Heterogeneity indicators for the outcome of "clinical efficacy treated for more than 5 weeks" in the studies considering renzapride compared to placebo therapy in IBS patients

A

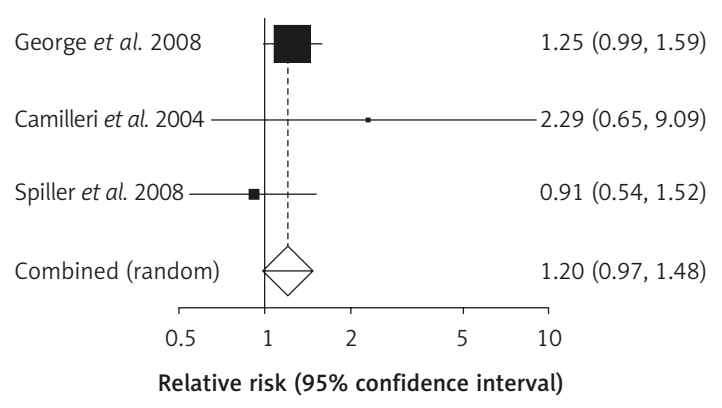

B

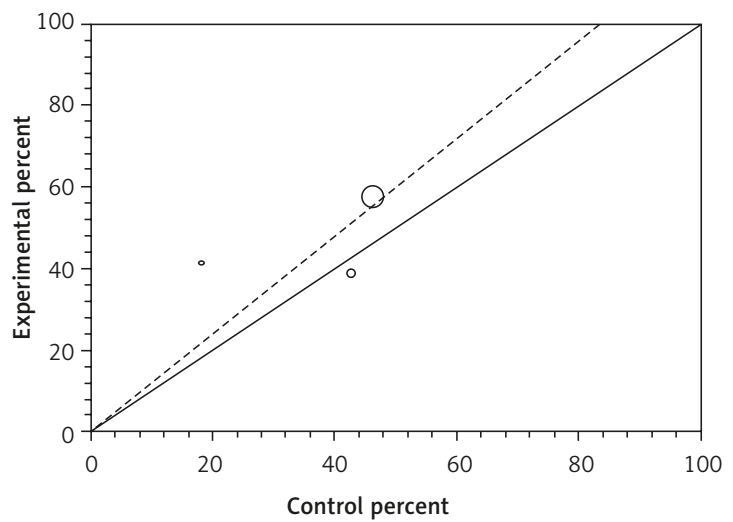

Figure 5. A - Individual and pooled relative risk for the outcome of "clinical efficacy treated for 5 weeks or less" in the studies considering renzapride $4 \mathrm{mg}$ daily compared to placebo therapy in IBS patients. B - Heterogeneity indicators for the outcome of "clinical efficacy treated for 5 weeks or less" in the studies considering renzapride $4 \mathrm{mg}$ daily compared to placebo therapy in IBS patients

A

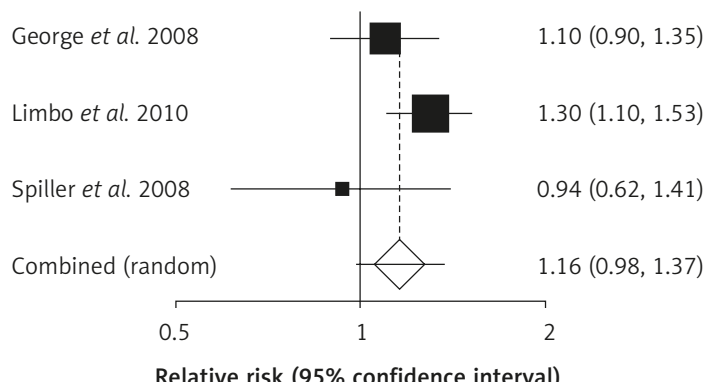

B

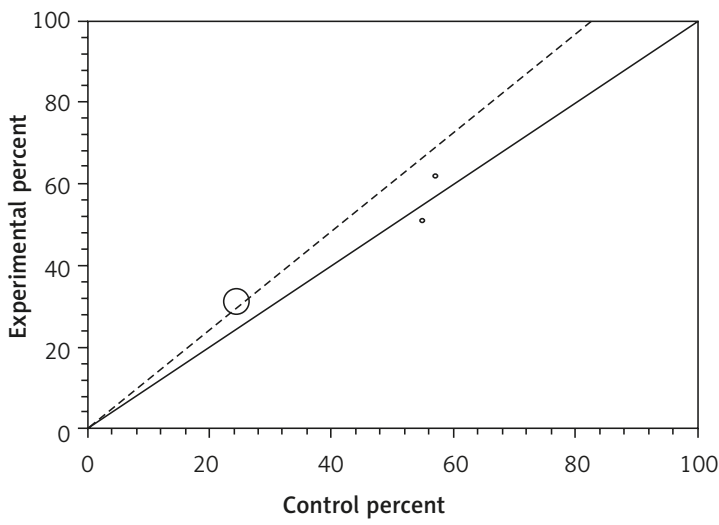

Figure 6. A - Individual and pooled relative risk for the outcome of "clinical efficacy treated for more than 5 weeks" in the studies considering renzapride $4 \mathrm{mg}$ daily compared to placebo therapy in IBS patients. B - Heterogeneity indicators for the outcome of "clinical efficacy treated for more than 5 weeks" in the studies considering renzapride $4 \mathrm{mg}$ daily compared to placebo therapy in IBS patients 
A

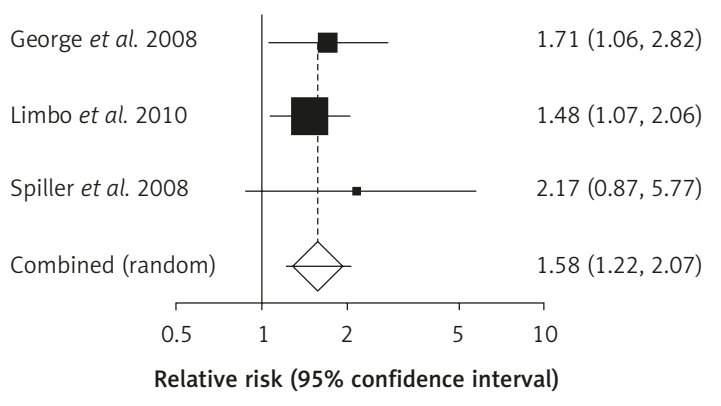

B

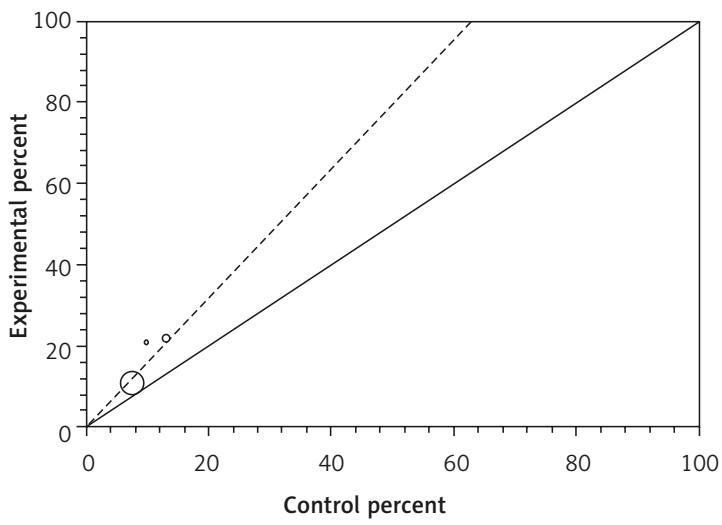

Figure 7. A - Individual and pooled relative risk for the outcome of "withdrawal" in the studies considering renzapride compared to placebo therapy in IBS patients. B - Heterogeneity indicators for the outcome of "withdrawal" in the studies considering renzapride compared to placebo therapy in IBS patients

The summary RR for abdominal pain in 3 trials $[19,20,22]$ was 1.37 with a $95 \% \mathrm{Cl}$ of $0.95-1.98$ and a non-significant RR ( $p=0.09)$. The Cochrane $\mathrm{Q}$ test for heterogeneity indicated that the studies are homogeneous $(p=0.51)$ and could be combined but because of few included studies the random effects for individual and summary of RR was applied.

The summary RR for constipation (aggravated) in 2 trials $[20,22]$ was 0.98 with a $95 \% \mathrm{Cl}$ of $0.55-1.74$ and a non-significant RR $(p=0.94)$. The Cochrane $\mathrm{Q}$ test for heterogeneity indicated that the studies are homogeneous ( $p=0.98)$ and could be combined but because of few included studies the random effects for individual and summary of RR was applied.

The summary RR for nausea in three trials [19, 21,22 ] was 1.27 with a $95 \% \mathrm{Cl}$ of $0.76-2.11$ and a non-significant RR $(p=0.36)$. The Cochrane $Q$ test for heterogeneity indicated that the studies are homogeneous $(p=0.27)$ and could be combined but because of few included studies the random effects for individual and summary of RR was applied.

The summary RR for dyspepsia (gas) in two trials $[21,22]$ was 0.94 with a $95 \% \mathrm{Cl}$ of $0.45-1.96$ and a non-significant RR $(p=0.88)$. The Cochrane $Q$ test for heterogeneity indicated that the studies are homogeneous $(p=0.96)$ and could be combined but because of few included studies the random effects for individual and summary of RR was applied.

The summary RR for vomiting in 4 trials [19-22] was 1.2 with a $95 \% \mathrm{Cl}$ of $0.74-1.95$ and a non-significant RR $(p=0.47)$. The Cochrane $\mathrm{Q}$ test for heterogeneity indicated that the studies are homogeneous ( $p=0.75$ ) and could be combined; thus the fixed effects for individual and summary of RR was applied (Table IV).

\section{Withdrawal due to adverse effect, non-compliance and lack of efficacy with renzapride compared to placebo therapy in irritable bowel syndrome patients}

The summary of RR for withdrawal in IBS patients in 3 included trials comparing renzapride to placebo $[19,20,22]$ was 1.58 with $95 \% \mathrm{Cl}=1.26$ to 2.07 ( $p=0.0007$, Figure $7 \mathrm{~A})$. The Cochrane $\mathrm{Q}$ test for heterogeneity indicated that the studies are not heterogeneous ( $p=0.73$, Figure 7 B) and could be combined but because of few included studies, the random effects for individual and summary of RR was applied. Regression of normalized effect vs. precision for all included studies for withdrawal in IBS patients receiving renzapride vs. placebo therapy could not be calculated because of too few strata.

\section{Discussion}

This meta-analysis included a total of 2528 C-IBS and non C-, non D-IBS patients according to the mentioned inclusion criteria from randomized placebo-controlled clinical trials. In all studies, the criteria for diagnosis of IBS patients was based on Rome criteria. Regarding the statistical analysis, the present results confirm that renzapride has no significant advantage over placebo in relieving symptoms in IBS patients. Regarding the prevalence and high burden of IBS, developing novel therapeutic agents for this syndrome is of great value. To reach a convincing conclusion on effectiveness of each new suggested drug, assessing clinical trials in which drug was compared to placebo could be clarifying. We analyzed data for efficacy twice based on duration of treatment. The results demonstrated that increasing the duration of treatment (more than 5 weeks) does not influence the efficacy of renzapride compared to placebo. 
Several systematic reviews have assessed the efficacy of $5 \mathrm{HT}$ receptor modulators in IBS patients. Ford et al. performed a systematic review on the efficacy of known 5-HT3 antagonists and 5-HT4 agonists in IBS in 2009 [17]. They conducted their meta-analysis by reviewing placebo-controlled clinical trials up to the year 2008. However, our meta-analysis included a 12-week double-blind, randomized, placebo-controlled clinical trial by Lembo et al. [19] in 2010, in which a total of 1798 female IBS patients were included for both efficacy and safety analysis. As in the present meta-analysis, Ford et al. confirmed that renzapride and cisapride were not more effective than placebo in IBS patients. Another group of researchers also evaluated the efficacy of mixed $5 \mathrm{HT} 3$ antagonists/5HT4 agonists (cisapride and renzapride) in IBS patients [22] and found that renzapride in doses of $1 \mathrm{mg}$ and $2 \mathrm{mg}$ was not effective in relieving IBS symptoms, which further supports the present meta-analysis. They demonstrated that a dose of $4 \mathrm{mg}$ was significantly more effective than placebo. For clarification, we analyzed the clinical efficacy of renzapride $4 \mathrm{mg}$ separately in comparison to placebo for 5 weeks or less, and more than 5 weeks treatment durations. Although differences were not statistically significant, the results were clinically important and significant for both treatment durations. Therefore, these results could be considered for renzapride $4 \mathrm{mg}$ while more trials are necessary to conclude the effectiveness of this novel drug more precisely. Several adverse effects have been reported for renzapride that were mainly in the Gl tract. By analyzing the reported adverse effects, we found that there was no statistically significant difference between renzapride and placebo, except in the occurrence of diarrhea, which was higher in patients receiving renzapride. In addition, renzapride caused more withdrawals due to adverse effects and/or low efficacy in patients. In the same study, no significant difference was observed between renzapride and placebo for occurrence of adverse effects in patients [23].

One of the limitations of this meta-analysis is the differences in the characteristics of involved patients (age, sex, life style and compliance), which is inevitable and results from deficits of included trials. In addition, included trials had different duration of treatment and endpoints. Treatment durations ranged from 2 weeks [21] to 12 weeks $[19,22]$. Therefore, to avoid heterogeneity, we divided our data into two groups according to treatment duration and time of reporting the results ( 5 weeks or less, and more than 5 weeks), although there were few data in each group. In these 4 assessed clinical trials, 3 doses of renzapride were used in patients (1, 2, and $4 \mathrm{mg}$ daily). We did not observe any significant difference be- tween renzapride and placebo, except for $4 \mathrm{mg}$, which in comparison to placebo had a clinically significant difference. To have more reliable results on the efficacy of renzapride and to find a possible dose-dependent effect, we analyzed data based on renzapride dose and compared the $4 \mathrm{mg}$ group to placebo separately. But, similar to duration of treatment, there were few patients in each group. In one of the trials the experimented population members were female [19] and in other three studies the authors reported better efficacy of renzapride in females than males [20-22]. Therefore to have a bigger population of patients with more homogeneity in sex and age to reach a more powerful conclusion, further clinical trials are essential. Although the results of this meta-analysis do not confirm the significant effectiveness of renzapride in IBS patients, regarding the acceptable quality score of included trials and lack of other suitable choices, it can still be recommended in C-IBS patients due to its accelerating effects on GI motility.

In conclusion, renzapride is not only superior to placebo in relieving IBS symptoms (abdominal pain and discomfort) but also causes significant incidence of diarrhea and drop-outs due to adverse effects in patients. This means that this drug might be a cost burden to patients without providing good efficacy or advantages.

\section{Acknowledgments}

This paper is the outcome of an in-house financially non-supported study. The authors have no competing interests. The authors thank the National Elite Foundation and INSF for supporting the first author's postdoc program.

\section{References}

1. Spiller R, Aziz Q, Creed F, et al. Guidelines on the irritable bowel syndrome: mechanisms and practical management. Gut 2007; 56: 1770-98.

2. Mahvi-Shirazi M, Fathi-Ashtiani A, Rasoolzade-Tabatabaei SK, Amini M. Irritable bowel syndrome treatment: cognitive behavioral therapy versus medical treatment. Arch Med Sci 2012; 8: 123-9.

3. Darvish-Damavandi M, Nikfar S, Abdollahi M. A systematic review of efficacy and tolerability of mebeverine in irritable bowel syndrome. World J Gastroenterol 2010; 16: 547-53.

4. Rahimi R, Nikfar S, Rezaie A, Abdollahi M. Efficacy of tricyclic antidepressants in irritable bowel syndrome: a meta-analysis. World J Gastroenterol 2009; 15: 1548-53.

5. Rahimi R, Nikfar S, Abdollahi M. Selective serotonin reuptake inhibitors for the management of irritable bowel syndrome: a meta-analysis of randomized controlled trials. Arch Med Sci 2008; 4: 71-6.

6. Rezaie A, Nikfar S, Abdollahi M. The place of antibiotics in management of irritable bowel syndrome: a systematic review and meta-analysis. Arch Med Sci 2010; 6: 49-55.

7. Nikfar S, Rahimi R, Rahimi F, Derakhshani S, Abdollahi M. Efficacy of probiotics in irritable bowel syndrome: a me- 
ta-analysis of randomized, controlled trials. Dis Colon Rectum 2008; 51: 1775-80.

8. Rahimi R, Abdollahi M. Herbal medicines for the management of irritable bowel syndrome: a comprehensive review. World J Gastroenterol 2012; 18: 589-600.

9. Stankiewicz W, Rongies W, Bodera P, et al. Immunoregulatory disorders in irritable bowel syndrome. Centr Eur J Immunol 2011; 36: 267-74.

10. Atkinson W, Lockhart S, Whorwell PJ, Keevil B, Houghton LA. Altered 5-hydroxytryptamine signaling in patients with constipation- and diarrhea-predominant irritable bowel syndrome. Gastroenterology 2006; 130: 34-43.

11. Talley NJ. 5-hydroxytryptamine agonists and antagonists in the modulation of gastrointestinal motility and sensation: clinical implications. Aliment Pharmacol Ther 1992; 6: 273-89

12. Mozaffari S, Nikfar S, Abdollahi M. Metabolic and toxicological considerations for the latest drugs used to treat irritable bowel syndrome. Expert Opin Drug Metab Toxicol 2013; 9: 403-21.

13. Meyers NL, Hickling RI. Pharmacology and metabolism of renzapride: a novel therapeutic agent for the potential treatment of irritable bowel syndrome. Drugs R D 2008; 9: 37-63.

14. Meyers NL, Hickling RI. The cardiovascular safety profile of renzapride, a novel treatment for irritable bowel syndrome. J Int Med Res 2007; 35: 848-66.

15. Tack J, Middleton SJ, Horne MC, et al. Pilot study of the efficacy of renzapride on gastrointestinal motility and symptoms in patients with constipation-predominant irritable bowel syndrome. Aliment Pharmacol Ther 2006; 23: 1655-65.

16. Scarpellini E, Tack J. Renzapride: a new drug for the treatment of constipation in the irritable bowel syndrome. Expert Opin Investig Drugs 2008; 17: 1663-70.

17. Ford AC, Brandt LJ, Young C, Chey WD, Foxx-Orenstein AE, Moayyedi P. Efficacy of 5-HT3 antagonists and 5-HT4 agonists in irritable bowel syndrome: systematic review and meta-analysis. Am J Gastroenterol 2009; 104: 1831-43.

18. Jadad A. Randomised controlled trials. London, BMJ Books 1998.

19. Lembo AJ, Cremonini F, Meyers N, Hickling R. Clinical trial: renzapride treatment of women with irritable bowel syndrome and constipation - a double-blind, randomized, placebo-controlled, study. Aliment Pharmacol Ther 2010; 31: 979-90.

20. Spiller RC, Meyers NL, Hickling RI. Identification of patients with non-D, non-C irritable bowel syndrome and treatment with renzapride: an exploratory, multicenter, randomized, double-blind, placebo-controlled clinical trial. Dig Dis Sci 2008; 53: 3191-200.

21. Camilleri M, McKinzie S, Fox J, et al. Effect of renzapride on transit in constipation-predominant irritable bowel syndrome. Clin Gastroenterol Hepatol 2004; 2: 895-904.

22. George AM, Meyers NL, Hickling RI. Clinical trial: renzapride therapy for constipation-predominant irritable bowel syndrome: multicentre, randomized, placebocontrolled, double-blind study in primary healthcare setting. Aliment Pharmacol Ther 2008; 27: 830-7.

23. He WR, Zhang FC, Liang LX. Mixed $5-\mathrm{HT}_{3}$ antagonists/5- $\mathrm{HT}_{4}$ agonists for irritable bowel syndrome: a systematic review. World Chin J Digestol 2011; 19: 3277-83. 